\title{
The Pathophysiology of Ischemic Stroke Studied by Radionuclide Imaging
}

\author{
Wolf-Dieter Heiss \\ Max Planck Institute for Metabolism Research, Cologne, Germany
}

Article Info

\section{Article Notes}

Received: October 11, 2016

Accepted: November 03, 2016

\section{*Correspondence:}

Prof. Dr. W.-D. Heiss

Max Planck Institute for Metabolism Research,

Gleueler Str. 50, 50931 Köln, Germany, Telephone: 49-221-4726220; Fax: 49-221-4726-349; Email: wdh@nf.mpg.de

(c) 2016 Heiss WD. This article is distributed under the terms of the Creative Commons Attribution 4.0 International License

\section{Keywords:}

SPECT

PET

Stroke

Cerebral Ischemia

Penumbra

Infarction

Reperfusion

Hemodynamic reserve

neuroinflammation

Diaschisis

Functional Activation

\section{ABSTRACT}

Ischemic stroke is caused by interruption or significant impairment of blood supply to the brain, which leads to a cascade of metabolic and molecular alterations resulting in functional disturbance and morphological damage. The changes in regional cerebral blood flow and in regional metabolism can be assessed by radionuclide imaging, especially single photon emission tomography (SPECT) and positron emission tomography (PET). SPECT and PET have broadened our understanding of flow and metabolic thresholds critical for maintenance of brain function and morphology: PET was essential in the transfer of the concept of the penumbra to clinical stroke and thereby had a great impact on developing treatment strategies. Receptor-ligands can be applied as early markers of irreversible neuronal damage and can predict the size of the final infarcts, which is important for decisions of invasive therapy in large ("malignant") infarction. With SPECT and PET the reserve capacity of blood supply can be tested in obstructive arteriosclerosis, which is essential for planning interventions. The effect of a stroke on surrounding and contralateral primarily not-affected tissue can be investigated helping to understand symptoms caused by disturbance in functional networks. Activation studies are useful to demonstrate alternative pathways to compensate for lesions and to test the effect of rehabilitative therapy. Radioisotope studies help to detect neuroinflammation and its effect on extension of tissue damage. Despite the limitations of broad clinical application of radionuclide imaging, this technology has a great impact on research in cerebrovascular diseases and still has various applications in the management of stroke. In this short review the contributions of PET- and SPECT-studies to the understanding of the pathophysiology of ischemic stroke are described.

\section{Introduction}

The energy demands of the nervous tissue are very high and therefore sufficient blood supply to the brain must be maintained consistently. A normal adult male's brain containing approx. 130 billion neurons ( 21.5 billion in the neocortex $)^{1}$ comprises only 2 $\%$ of total body mass, yet consumes at rest approximately $20 \%$ of the body's total basal oxygen consumption supplied by $16 \%$ of the cardiac blood output. The brain's oxygen consumption is almost entirely for the oxidative metabolism of glucose, which in normal physiological conditions is the almost exclusive substrate for the brain's energy metabolism. Energy metabolism by functional activation is due mostly to stimulation of the $\mathrm{Na}+\mathrm{K}+-\mathrm{ATPase}$ activity to restore the ionic gradients across the cell membrane and the membrane potentials that were degraded by the spike activity and is rather high compared to the demand of neuronal cell bodies ${ }^{2}$. Overall, $87 \%$ of the total energy consumed is required by signalling, 
mainly action potential propagation and postsynaptic ion fluxes, and only $13 \%$ is expended in maintaining membrane resting potential ${ }^{3}$.

Circulatory disturbances and insufficient blood supply trigger a complex deleterious cascade of biochemical and molecular events finally leading to ischemic cell death (review in Hossmann 20094). The result - ischemic stroke - is a major health burden for society: in 2000 there were 15.3 million strokes world-wide, 5.5 million resulted in death $^{5}$. But cerebrovascular disease CVD accounts not only for $10 \%$ of all deaths; it is the leading cause of disability in patients surviving the insult ${ }^{6}$. Radionuclide imaging detects the pathophysiological changes occurring in ischemic stroke and therefore may help in treatment decisions and may guide the development of new therapeutic strategies.

\section{Detection of the ischemic lesion}

Localization and extension of lesions as a result of defects in blood supply can be assessed by SPECT studies of perfusion and by PET studies of flow and / or metabolism. Both techniques have been shown to be more sensitive than $\mathrm{x}$-ray computed tomography (CT) for detecting both the presence and extent of infarction ${ }^{7,8}$. In the first 8 hours after stroke SPECT was positive in $90 \%$ and sensitivities of 61 to $74 \%$ and specificities of 88 to $98 \%$ were reported ${ }^{9}$. Early PET studies already showed discrepancies between flow and glucose uptake in ischemic areas suggesting anaerobic glycolysis ${ }^{10}$ and with determination of oxygen extraction fraction (OEF) permitted to follow the development of infarction within $48 \mathrm{~h}$ after the ictus ${ }^{11}$. Increased perfusion was observed during this period despite irreversible tissue damage, which was termed "luxury perfusion" ${ }^{12}$; in later stages relative hyperemia can be observed in the core as well as in the surrounding of infarcts. Transient ischemic attacks (TIAs) can be differentiated from ischemic strokes within $6 \mathrm{~h}$ of symptoms onset in SPECT by count rate densities of $70 \%$ compared to the contralateral side (perfusion in stroke tissue $35-60 \%$ of contralateral values $)^{13}$ and in PET by significantly decreased CBF but increased OEF in affected regions ${ }^{14}$.

SPECT findings correlated with the severity of neurological deficits and clinical outcome: early severe hypoperfusion predicted poor outcome, especially when the volume of perfusion deficit was considered, better than neurological deficit scores and correlated to infarct size on late CTs (review in ${ }^{15}$ ). Overall, SPECT had a significant added predictive value to neurological scores, although it was not as reliable as PET, by which a classification of patients according to patterns of perfusion and oxygen consumption changes within 5-8 $\mathrm{h}$ of stroke onset predicted good or poor outcome and indicated critical states of ischemia amenable to therapy ${ }^{16}$.
The concept of the ischemic penumbra and identification by imaging

Experimental work on the ischemic flow thresholds of brain tissue demonstrated the existence of two critical levels of decreased perfusion: first, a level representing the flow threshold for reversible functional failure (functional threshold); second, a lower threshold below which irreversible membrane failure and morphological damage occur. The range of perfusion values between those limits was called the "ischemic penumbra,"17 which was characterized by the potential for functional recovery without morphological damage, provided that local blood flow can be reestablished at a sufficient level. Whereas neuronal function is impaired immediately when blood flow drops below the threshold, the development of irreversible morphological damage is time dependent. This interaction of severity and duration of ischemia in the development of irreversible cell damage was established in simultaneous recordings of cortical neuronal activity and local blood flow ${ }^{18}$. These results complement the concept of ischemic penumbra: the potential for postischemic recovery of functionally impaired cells is determined not only by the level of residual flow in the ischemic phase but also by the duration of the flow disturbance.

Early PET studies in stroke have identified various tissue compartments within a brain territory compromised by ischemia ${ }^{19-22}$. Tissue with regional cerebral blood flow $(\mathrm{rCBF})<12 \mathrm{ml} / 100 \mathrm{~g} / \mathrm{min}$ or regional metabolic rate for oxygen $\left(\mathrm{rCMRO}_{2)}<65 \mu \mathrm{mol} / 100 \mathrm{~g} / \mathrm{min}\right.$ at the time of measurement (usually several hours after stroke) was found to be infarcted on late CTs. Relatively preserved $\mathrm{CMRO}_{2}$ was an indicator of maintained neuronal integrity in regions with CBF reduced to $12-22 \mathrm{ml} / 100 \mathrm{~g} / \mathrm{min}$. This pattern, coined misery perfusion ${ }^{20}$ served as a definition of the penumbra that is characterised as the area of an increased oxygen extraction fraction (up to $>80 \%$ from the normal value of approximately $40 \%$ ). PET studies allow the classification of three regions within the disturbed vascular territory: the core of ischemia with a flow $<12$ $\mathrm{ml} / 100 \mathrm{~g} / \mathrm{min}$ usually showing a transition into necrosis; a penumbra region with a flow between 12 and $22 \mathrm{ml} / 100$ $\mathrm{g} / \mathrm{min}$ of still viable tissue but with uncertain chances for infarction or recovery; and a hypoperfused area $(>22$ $\mathrm{ml} / 100 \mathrm{~g} / \mathrm{min}$ ) not primarily damaged by the lack of blood supply. The extent of the penumbra and its conversion into infarction is a dynamic process, and irreversible damage spreads from the core of ischemia to its border. This can be followed directly with advanced PET equipment, by which changes in the physiologic variables were studied after occlusion of the middle cerebral artery (MCA) in baboons and cats (review in ${ }^{23-25}$ ). 


\section{Non-invasive imaging of the penumbra}

Measurement of blood flow values and determination of oxygen extraction fraction by ${ }^{15} \mathrm{O}$-PET require arterial blood sampling. A marker of neuronal integrity is needed that can identify irreversibly damaged tissue irrespective of the time elapsed since the vascular attack, which also does not require arterial blood sampling. The central benzodiazepine receptor ligand flumazenil (FMZ) binds to the GABA receptor abundant in the cerebral cortex. These receptors are sensitive to ischemic damage and can therefore identify early neuronal loss. With this tracer the pathophysiological changes early after ischemic stroke could be accurately specified: $55 \%$ of the volume of the final infarct had FMZ uptake indicative of infarction in the first hours after stroke; $21 \%$ of the final infarct had flow below $14 \mathrm{ml} / 100 \mathrm{~g} / \mathrm{min}$, but FMZ uptake above the critical value, thereby indicating penumbra tissue ${ }^{23}$.

MR studies using diffusion and perfusion imaging might provide a differentiation between the core and the penumbra: the early diffusion weighted imaging (DWI) lesion might define the ischemic core and adjacent critically hypoperfused tissue might be identified with perfusion-weighted imaging (PWI) ${ }^{26}$. Therefore, brain regions with hypoperfusion assessed by PWI but without restricted diffusion (PWI / DWI mismatch) were assumed to represent the penumbra. This surrogate definition of the penumbra has several uncertainties ${ }^{27}$ :

Several studies were performed in order to validate mismatch as a surrogate of penumbra on PET-derived discrimination of irreversibly damaged, critically perfused "at risk" and oligemic "not at risk" tissue. The studies demonstrated that the DWI lesion predicts more or less the finally infarcted tissue ${ }^{28}$, but contains up to $25 \%$ false positive, i.e. surviving tissue. The inaccuracy in defining the penumbra with PW/DWI mismatch is mainly related to PW data acquisition, which is a complex process, and the parameters used to estimate perfusion are variable and somewhat arbitrary. As a consequence, perfusion lesion size differs markedly depending on the parameters calculated $^{29}$ and usually is overestimated. Time to peak delays of 4 and $6 \mathrm{sec}$ reliably identified hypoperfused and excluded normoperfused tissue but overestimated the volume of critically perfused but salvageable tissue, i.e. the penumbra ${ }^{30}$ : The mismatch volume in PW / DWI as conventionally calculated therefore does not reliably reflect misery perfusion, i.e. the penumbra as defined by PET.

\section{Detection of hypoxic tissue}

Markers of hypoxic tissue were also tested with respect to their capacity to identify penumbral tissue. The nitroimidazole derivative misonidazole, labeled with ${ }^{18}$ fluor (FMISO), was first used by Yeh et al $1994^{31}$ to image cerebral ischemia in humans and revealed increased uptake surrounding a zone of absent activity. This zone of high activity had disappeared when imaging was performed during the chronic phase, indicating that the hypoxic tissue had either infarcted or recovered. This pattern and time course was confirmed in a larger study ${ }^{32}$. Tissue with increased FMISO uptake was generally distributed over the periphery of the infarct, identified on the coregistered late CT, but extended into normal tissue adjacent to the infarct in a few cases. The amount of hypoxic tissue detected by FMISO declined as a function of time after stroke onset. The proportions of hypoxic tissue that infarcted or survived varied between patients. Within 6 hours of stroke onset about $90 \%$ of the FMISO positive region is included in the final infarct but this percentage is reduced later on ${ }^{33}$.Volume of initially affected tissue and initial severity of neurologic deficits as well as proportion of initially affected tissue progressing to infarction and neurological deterioration during the first week after stroke are correlated ${ }^{34}$. As high FMISO uptake in these areas fulfills the definition of the penumbra, topography of FMISO trapping was used to generate a so called "penumbragram"35.

\section{PET as a surrogate marker for treatment efficiency}

The effect of the only approved conservative therapy for acute ischemic stroke was established also in imaging studies, in which reperfusion to penumbral tissue was followed by improvement in neurological deficits: Reperfusion was significantly increased in rtPA treated patients compared to controls ${ }^{36}$. The volume of tissue salvaged by reperfusion was established in a study in which $\mathrm{CBF}$, as determined by $\mathrm{H}_{2}{ }^{15} \mathrm{O}$-PET within 3 hours of stroke onset, was compared with the volume of infarction determined on MRI 3 weeks after the ictus ${ }^{37}$. The percentage of initially critically ischemic voxels (i.e. with a flow below the threshold of $12 \mathrm{ml} / 100 \mathrm{~g} / \mathrm{min}$ ) that became reperfused at almost normal levels clearly predicted the degree of clinical improvement achieved within 3 weeks. Overall, only $22.7 \%$ of the grey matter that was initially perfused at rates below the conventional threshold of critical ischemia became necrotic after thrombolytic therapy in this small sample of 12 patients. This means, that a considerable portion of the critically hypoperfused tissue was probably salvaged by the reperfusion therapy. Another PET study in 11 patients $^{38}$ indicated that hypoperfused tissue could benefit from reperfusion only as long as cortical flumazenil binding was not reduced to or below 3.4 times the mean uptake in white matter. This marker of neuronal integrity can therefore serve as an indicator for irreversibly damaged tissue that is not amenable to treatment.

\section{PET for prediction of "malignant infarction"}

Malignant brain infarcts develop in about $10 \%$ of patients with ischemic stroke in the middle cerebral artery (MCA) territory. Invasive treatment strategies, especially 
decompressive hemicraniectomy might improve mortality and morbidity of these patients ${ }^{39}$. The selection of patients who can benefit and the determination of the best time for these interventions however are still controversial and require a better assessment of the extent of irreversible ischemic damage.

In 34 patients with ischemic changes of $>50 \%$ of the MCA territory in early cerebral CT scans PET was performed with 11C-FMZ to assess CBF and irreversible neuronal damage. Thereafter, probes for microdialysis and for measurement of intercranial pressure and tissue oxygen pressure were placed into the ipsilateral frontal lobe ${ }^{40}$. PET measurements within 24 hours after stroke showed larger volumes of ischemic core (mean, 144.5 versus $62.2 \mathrm{~cm} 3$ ) and larger volumes of irreversible neuronal damage (157.9 versus $47.0 \mathrm{~cm} 3$ ) in patients with malignant course (ie, edema formation with midline shift) than in patients with benign course. Mean cerebral blood flow values within the ischemic core were significantly lower and the volume of the ischemic penumbra was smaller in the malignant than in the benign group. In patients with malignant course, cerebral perfusion pressure dropped to $<50$ to $60 \mathrm{~mm} \mathrm{Hg}$ 22 to 72 hours (mean, 52.0 hours) after onset of symptoms; subsequently, $\mathrm{PtO}_{2}$ dropped and glutamate increased, indicating secondary ischemia.

\section{Microglia activation as an indicator of inflammation}

Microglia constitutes up to $10 \%$ of the total cell population of the brain. As resident macrophages of the central nervous system, microglia phagocytose cellular debris, present foreign antigens, and are sensors of pathological events including ischemia ${ }^{41}$. Microglia changes from a resting to an activated state in response to central nervous system insults that stimulate them to function as phagocytes. Several radioligands have been developed to image the activation of microglia in experimental models and in various diseases of the central nervous system ${ }^{42}$.

Many histological studies have documented the presence of activated microglia in the ischemic brain after stroke in humans. Abundant reactive microglia/macrophages have been found in the ischemic core within 1 to 2 days after ischemic infarction. Over time, they extended from the ischemic core into the peri-infarct zones $^{43,44}$. In several imaging studies increased binding of the mitochondrial benzodiazepine receptor antagonist 11C-PK11195 was observed around the outer border of ischemic lesions after several days, but also in areas distant from the lesion (review in ${ }^{42}$ ). Increased tracer binding was also detected in the thalamus ipsilateral to the stroke and in the subcortical white matter tracts. Using diffusion tensor imaging-based definitions of the pyramidal tract, a prospective controlled study $^{45}$ demonstrated that microglia activation occurs along the pyramidal tract anterograde to the lesion only in those patients with acute subcortical stroke, where the pyramidal tract was affected. These anterograde tract portions will undergo Wallerian degeneration in the weeks and months after stroke.

\section{Hemodynamic and Metabolic Reserve}

Patients with arterial occlusive disease are protected against ischemic episodes to a certain extent by compensatory mechanisms, which help to prevent ischemia when perfusion pressure drops. This condition, studied by PET using ${ }^{15}$ O-labelled tracers ${ }^{46}$ in patients with uni- or bilateral carotid artery disease, is indicated by regional vasodilatation manifesting itself as a focal increase in cerebral blood volume (CBV) in the supply territory of the occluded artery. Since a critical CBV distinguishing occluded from patent arterial territories could not be defined, the ratio of CBF to CBV was used as an indicator of local perfusion pressure. By calculating CBF / CBV (normal value 10), territories of patent carotids, of unilateral occlusion, of occlusion with contralateral stenoses, and of bilateral occlusions could be discriminated. Therefore, this ratio representing the reciprocal of the local mean vascular transit time is a measure of the perfusion reserve: the lower its value (from a normal value of 10 down to 5.5), the lower the flow velocity and the longer the residence time. The lowest ratios were found in patients with symptoms suggestive of a hemodynamic rather than a thrombotic component to their ischemia, in which maximal vasodilatation and low regional perfusion pressure may be expected. When the perfusion reserve is exhausted (i.e. at maximal vasodilatation), any further decrease in arterial input pressure produces a proportional decrease in both $\mathrm{CBF}$ and the $\mathrm{CBF} / \mathrm{CBV}$ ratio. In this condition of hemodynamic decompensation, the brain must draw upon the oxygen-carriage reserve to prevent energy failure and loss of function, as evidenced by an increase in the OER, from the normal value of $40 \%$ to up to $85 \%{ }^{46-48}$. On the basis of such PET measurements, it is therefore possible to discriminate patients with impaired hemodynamics only, as well as to quantify the impairment of perfusion reserve, using the CBF / CBV ratio.

\section{Deactivation of Remote Tissue (Diaschisis)}

Even the earliest PET studies of ischemic brain lesions ${ }^{49}$ revealed reduction of metabolism and blood flow exceeding the extent of morphologically damaged tissue - a regular finding since then with other functional imaging modalities as well, such as SPECT. The most conspicuous effect was a reduction of CBF and metabolism in the contralateral cerebellum, called "crossed cerebellar diaschisis"50, occurring immediately after a stroke and persisting permanently in patients suffering from lesions involving the cortico-pontine-cerebellar pathways but is reversed by successful reperfusion therapy ${ }^{51}$. This CCD was 
obviously due to some neuronally mediated effect, since a primary vascular cause could be excluded on account of the remote vascular territory. Further remote effects included reductions of $\mathrm{CBF}$ and metabolism in the ipsilateral cortex and basal ganglia. Clinical symptoms that are difficult to relate to the infarct proper can often be explained by remote effects, and the severity of the metabolic disorder appears to be inversely related to later functional recovery ${ }^{7}$.

\section{Complex activation studies}

Regional cerebral metabolism and blood flow are dependent on the functional state of the brain tissue. A direct coupling of functional activity and focal blood flow could be demonstrated already by the 133 Xenon clearance method for measurement of regional cerebral blood flow ${ }^{52}$, by which two dimensional cortical activation patterns for various tasks including speech and memory could be obtained. With the advance of PET three-dimensional regional activation studies became feasible in healthy controls and in patients with various CNS disorders (review $^{53}$ ).

In the brain of healthy right-handers and the majority of left-handers language is a function of the left, dominant hemisphere. Language areas active in the dominant hemisphere (e.g. Broca's Area) suppress activity in homologous areas of the non-dominant hemisphere (transcallosal inhibition). The existence of these inhibitory mechanisms has been deducted from imaging studies in patients with brain lesions and has directly been demonstrated in normal subjects using imaging-guided repetitive transcranial magnetic stimulation (rTMS) ${ }^{54}$ A unilateral and focal brain lesion (such as a stroke) to language areas of the dominant hemisphere does not only reduce activity in the affected hemisphere thus causing aphasia, but also releases activity in the unaffected hemisphere, via interruption of those transcallosal fibres (transcallosal disinhibition) ${ }^{55}$. This activity of brain regions in the non-dominant hemisphere in the first days and weeks after a stroke has repeatedly been demonstrated in sequential brain imaging studies ${ }^{56}$. In the following weeks and months of recovery brain activation shifts back to the dominant hemisphere. The extent of this backward shift to the dominant hemisphere varies from patient to patient and appears to be a major factor for successful recovery of language function ${ }^{57}$ in the acute and sub-acute phase. Results from a study in post-stroke aphasia shows that activity of the non-lesioned hemisphere is decreased by inhibitory rTMS. The shift of activation to the dominant hemisphere is related to an improvement in language function by speech therapy combined to inhibitory rTMS of the Broca homologous area of the non-dominant hemisphere ${ }^{58}$.

\section{Future perspectives: Multimodal imaging}

With new technical developments for integration of different modalities - e.g. MRI and PET - the investigations of various parameters (morphology, metabolism, blood flow and perfusion, molecular activities) can be performed simultaneously. Combining the functional metabolic values provided by PET in the picomolar range with the fast highresolution information from MRI in the micromolar range has already widespread applications in experimental research $^{59}$ and promising potential in humans ${ }^{60-62}$. The coregistration of various parameters improves quality of results: MR data are applied for correction for partial volume effects in small structures and dynamic information from MRI can be used for quantification of parametric values by PET.

Simultaneous multifunctional and multiparametric imaging might have a significant impact in stroke research: it guarantees exactly the same physiological state for comparative measurements of perfusion by PET and MRI; it permits differentiation of core and penumbra, demonstrates time-dependent growth of infarction and may be used to determine the optimal therapy for a certain time after the stroke; the vascular origin of the stroke can be detected by magnetic resonance angiography (MRA) and perfusion or oxy/deoxyhemoglobin (blood oxygen level dependent (BOLD) effect) changes can be related to the extent of oxygen deprivation (150) and hypoxia (FMISO) and to changes in metabolic markers (FDGPET, magnetic resonance spectroscopy (MRS) for lactate, choline, $\mathrm{N}$-acetylaspartate) - complimentary information important for therapeutic decisions. This high-resolution anatomic information is complemented by diffusion-based tractography, and can be related to activation or inhibition of neurotransmitter.

\section{References}

1. Pakkenberg B, Gundersen HJ. Neocortical neuron number in humans: effect of sex and age. J Comp Neurol. 1997;384:312-320

2. Sokoloff L. Energetics of functional activation in neural tissues. Neurochemical research. 1999;24:321-329

3. Laughlin SB, Attwell D. The metabolic cost of neural information: from fly eye to mammalian cortex. In: Frackowiak RSJ, Magistretti PJ, Shulman RG et al., eds. Neuroenergetics: Relevance for Functional Brain Imaging. Strasbourg: HFSP - Workshop XI, 2001:54-64

4. Hossmann KA. Pathophysiological basis of translational stroke research. Folia Neuropathol. 2009;47:213-227

5. WHO. Burden of Disease Statistics. World Health Organisation Geneva, Switzerland, 2008:http://www.who.int/healthinfo/bod/en/ index. html(assessed May 2, 2008)

6. Lopez AD, Mathers CD, Ezzati M, et al. Global and regional burden of disease and risk factors, 2001: systematic analysis of population health data. Lancet. 2006;367:1747-1757

7. Kushner M, Reivich M, Fieschi C, et al. Metabolic and clinical correlates of acute ischemic infarction. Neurology. 1987;37:1103-1110 
8. Raynaud C, Rancurel G, Tzourio N, et al. SPECT analysis of recent cerebral infarction. Stroke. 1989;20:192-204

9. Brass LM, Walovitch RC, Joseph JL, et al. The role of single photon emission computed tomography brain imaging with $99 \mathrm{mTc}$-bicisate in the localization and definition of mechanism of ischemic stroke. J Cereb Blood Flow Metab. 1994;14 Suppl 1:S91-98

10. Kuhl DE, Phelps ME, Kowell AP, et al. Effects of stroke on local cerebral metabolism and perfusion: mapping by emission computed tomography of 18FDG and 13NH3. Ann Neurol. 1980;8:47-60

11. Wise RJS, Bernardi S, Frackowiak RSJ, et al. Serial observations on the pathophysiology of acute stroke. The transition from ischaemia to infarction as reflected in regional oxygen extraction. Brain. 1983;106:197-222

12. Lassen NA. The luxury-perfusion syndrome and its possible relation to acute metabolic acidosis localized within the brain. Lancet. 1966;2:1113-1115

13. Berrouschot J, Barthel H, Hesse S, et al. Differentiation between transient ischemic attack and ischemic stroke within the first 6 hours after onset of symptoms by using 99m Tc-ECD-SPECT. J.Cereb.Blood Flow Metab. 1998;18:921-929

14. Powers WJ. Cerebral hemodynamics in ischemic cerebrovascular disease. Ann.Neurol. 1991;29:231-240

15. Ueda T. Can the penumbra be imaged using single-photon emission computed tomography? In: Donnan GA, Baron JC, Davis SM, Sharp FR, eds. The Ischemic Penumbra: CRC Press, 2007:165 - 175

16. Marchal G, Serrati C, Rioux P, et al. PET imaging of cerebral perfusion and oxygen consumption in acute ischaemic stroke: relation to outcome. Lancet. 1993;341:925-927

17. Astrup J, Siesjö BK, Symon L. Thresholds in cerebral ischemia - the ischemic penumbra. Stroke. 1981;12:723-725

18. Heiss WD, Rosner G. Functional recovery of cortical neurons as related to degree and duration of ischemia. Ann.Neurol. 1983;14:294-301

19. Ackerman RH, Correia JA, Alpert NM, et al. Positron imaging in ischemic stroke disease using compounds labeled with oxygen 15. Initial results of clinicophysiologic correlations. Arch.Neurol. 1981;38:537-543

20. Baron JC, Bousser MG, Comar D, et al. Noninvasive tomographic study of cerebral blood flow and oxygen metabolism in vivo. Potentials, limitations, and clinical applications in cerebral ischemic disorders. Eur.Neurol. 1981;20:273-284

21. Lenzi GL, Frackowiak RSJ, Jones T. Cerebral oxygen metabolism and blood flow in human cerebral ischemic infarction. J.Cereb.Blood Flow Metab. 1982;2:321-335

22. Powers WJ, Grubb RL, Jr., Darriet D, Raichle ME. Cerebral blood flow and cerebral metabolic rate of oxygen requirements for cerebral function and viability in humans. J.Cereb.Blood Flow Metab. 1985;5:600-608

23. Heiss WD. Ischemic penumbra: evidence from functional imaging in man. J.Cereb.Blood Flow Metab. 2000;20:1276-1293

24. Powers WJ, Zazulia AR. PET in Cerebrovascular Disease. PET Clin. 2010;5:83106

25. Heiss WD. David sherman lecture 2012: the role of positron emission tomography for translational research in stroke. Stroke. 2012;43:2520-2525

26. Barber PA, Darby DG, Desmond PM, et al. Prediction of stroke outcome with echoplanar perfusion-weighted and diffusion-weighted MRI. Neurology. 1998;51:418-426

27. Kidwell CS, Alger JR, Saver JL. Beyond mismatch: evolving paradigms in imaging the ischemic penumbra with multimodal magnetic resonance imaging. Stroke. 2003;34:2729-2735
28. Heiss WD, Sobesky J, Smekal U, et al. Probability of cortical infarction predicted by flumazenil binding and diffusion-weighted imaging signal intensity: a comparative positron emission tomography/ magnetic resonance imaging study in early ischemic stroke. Stroke. 2004;35:1892-1898

29. Kane I, Carpenter T, Chappell F, et al. Comparison of 10 different magnetic resonance perfusion imaging processing methods in acute ischemic stroke: effect on lesion size, proportion of patients with diffusion/perfusion mismatch, clinical scores, and radiologic outcomes. Stroke. 2007;38:3158-3164

30. Sobesky J, Weber OZ, Lehnhardt FG, et al. Does the mismatch match the penumbra? Magnetic resonance imaging and positron emission tomography in early ischemic stroke. Stroke. 2005;36:980-985

31. Yeh SH, Liu RS, Hu HH, et al. Ischemic penumbra in acute stroke: demonstration by PET with fluorine-18 fluoromisonidazole. J.Nucl. Med. 1994;35:205P

32. Read SJ, Hirano T, Abbott DF, et al. Identifying hypoxic tissue after acute ischemic stroke using PET and 18F-fluoromisonidazole. Neurology. 1998;51:1617-1621

33. Markus R, Reutens DC, Kazui S, et al. Topography and temporal evolution of hypoxic viable tissue identified by $18 \mathrm{~F}$-fluoromisonidazole positron emission tomography in humans after ischemic stroke. Stroke. 2003;34:2646-2652

34. Read SJ, Hirano T, Abbott DF, et al. The fate of hypoxic tissue on 18F-fluoromisonidazole PET after ischemic stroke. Ann.Neurol. 2000;48:228-235

35. Markus R, Donnan G, Kazui S, et al. Penumbral topography in human stroke: methodology and validation of the 'Penumbragram' Neuroimage. 2004;21:1252-1259

36. Grotta JC, Alexandrov AV. tPA-associated reperfusion after acute stroke demonstrated by SPECT. Stroke. 1998;29:429-432

37. Heiss WD, Grond M, Thiel A, et al. Tissue at risk of infarction rescued by early reperfusion: a positron emission tomography study in systemic recombinant tissue plasminogen activator thrombolysis of acute stroke. J.Cereb.Blood Flow Metab. 1998;18:1298-1307

38. Heiss WD, Kracht L, Grond M, et al. Early [ 11 C]Flumazenil/H 20 positron emission tomography predicts irreversible ischemic cortical damage in stroke patients receiving acute thrombolytic therapy. Stroke. 2000;31:366-369

39. Juttler E, Bosel J, Amiri H, et al. DESTINY II: DEcompressive Surgery for the Treatment of malignant INfarction of the middle cerebral arterY II. Int J Stroke. 2011;6:79-86

40. Dohmen C, Bosche B, Graf R, et al. Prediction of malignant course in MCA infarction by PET and microdialysis. Stroke. 2003;34:2152-2158

41. Hanisch UK, Kettenmann H. Microglia: active sensor and versatile effector cells in the normal and pathologic brain. Nat Neurosci. 2007;10:1387-1394

42. Thiel A, Heiss WD. Imaging of microglia activation in stroke. Stroke. 2011;42:507-512

43. Weinstein JR, Koerner IP, Moller T. Microglia in ischemic brain injury Future Neurol. 2010;5:227-246

44. Gerhard A, Schwarz J, Myers R, et al. Evolution of microglial activation in patients after ischemic stroke: a [11C](R)-PK11195 PET study. Neuroimage. 2005;24:591-595

45. Radlinska BA, Ghinani SA, Lyon P, et al. Multimodal microglia imaging of fiber tracts in acute subcortical stroke. Ann Neurol. 2009;66:825832

46. Gibbs JM, Wise RJ, Leenders KL, Jones T. Evaluation of cerebral perfusion reserve in patients with carotid-artery occlusion. Lancet. 1984;1:310-314 
47. Powers WJ, Press GA, Grubb RL, Jr. et al. The effect of hemodynamically significant carotid artery disease on the hemodynamic status of the cerebral circulation. Ann.Intern.Med. 1987;106:27-35

48. Sette G, Baron JC, Mazoyer B, et al. Local brain haemodynamics and oxygen metabolism in cerebrovascular disease. Positron emission tomography. Brain. 1989;112 ( Pt 4):931-951

49. Kuhl DE, Phelps ME, Kowell AP, et al. Effects of stroke on local cerebral metabolism and perfusion: Mapping by emission computed tomography of 18 FDG and 13 NH 3 Ann.Neurol. 1980;8:47-60

50. Baron JC, Bousser MG, Comar D, Castaigne P. "Crossed cerebellar diaschisis" in human supratentorial brain infarction. Trans.Am.Neurol. Assoc. 1980;105:459-461

51. Sobesky J, Thiel A, Ghaemi M, et al. Crossed cerebellar diaschisis in acute human stroke: a PET study of serial changes and response to supratentorial reperfusion. J.Cereb.Blood Flow Metab. 2005;25:1685-1691

52. Ingvar DH. Functional landscapes of the dominant hemisphere. Brain Res. 1976;107:181-197

53. Phelps ME, Mazziotta JC. Positron emission tomography: Human brain function and biochemistry. Science. 1985;228:799-809

54. Thiel A, Schumacher B, Wienhard K, et al. Direct demonstration of transcallosal disinhibition in language networks. J Cereb.Blood Flow Metab. 2006;26:1122-1127
55. Thiel A, Habedank B, Herholz K, et al. From the left to the right: How the brain compensates progressive loss of language function. Brain Lang. 2006;98:57-65

56. Saur D, Lange R, Baumgaertner A, et al. Dynamics of language reorganization after stroke. Brain. 2006;129:1371-1384

57. Heiss WD, Kessler J, Thiel A, et al. Differential capacity of left and right hemispheric areas for compensation of poststroke aphasia. Ann. Neurol. 1999;45:430-438

58. Thiel A, Hartmann A, Rubi-Fessen I, et al. Effects of noninvasive brain stimulation on language networks and recovery in early poststroke aphasia. Stroke. 2013;44:2240-2246

59. Wehrl HF, Judenhofer MS, Wiehr S, Pichler BJ. Pre-clinical PET/MR: technological advances and new perspectives in biomedical research. Eur J Nucl Med Mol Imaging. 2009;36 Suppl 1:S56-68

60. Heiss W-D. The potential of PET/MR for brain imaging. Eur J Nucl MEd Mol Imaging. 2009;36. Suppl 1:105-112

61. Sauter AW, Wehrl HF, Kolb A, et al. Combined PET/MRI: one step further in multimodality imaging. Trends Mol Med. 2010;16:508-515

62. Catana C, Drzezga A, Heiss WD, Rosen BR. PET/MRI for Neurologic Applications. J Nucl Med. 2012 